\title{
Religious coping and religiosity in patients with COPD following pulmonary rehabilitation
}

This article was published in the following Dove Press journal:

International Journal of COPD

\author{
Guilherme PF da Silva ${ }^{1,2}$ \\ Francisco AB Nascimento 1,3 \\ Tereza PM Macêdo' \\ Maria T Morano ${ }^{2,3}$ \\ Rafael Mesquita ${ }^{4}$ \\ Eanes DB Pereira ${ }^{1,3}$ \\ 'Department of Clinical Medicine, \\ Federal University of Ceará \\ (UFC), Fortaleza, ${ }^{2}$ Department of \\ Physiotherapy, University of Fortaleza \\ (UNIFOR), Fortaleza, ${ }^{3}$ Pulmonary \\ Rehabilitation Center, Hospital de \\ Messejana Dr Carlos Alberto Studart \\ Gomes, Fortaleza, ${ }^{4}$ Department of \\ Physiotherapy, Federal University of \\ Ceará (UFC), Fortaleza, Brazil
}

Correspondence: Eanes DB Pereira Department of Clinical Medicine, Federal University of Ceará (UFC), Fortaleza, Brazil

Tel +55 8534949000

Email eanes@fortalnet.com.br

\begin{abstract}
Background: Religious coping (RC) is defined as the use of behavioral and cognitive techniques in stressful life events in a multidimensional construct with positive and negative effects on outcomes, while religiosity is considered a use of individual beliefs, values, practices, and rituals related to faith. There is no evidence for the effects of pulmonary rehabilitation (PR) in RC and religiosity in patients with COPD. The aims of this study were 1) to compare RC and religiosity in patients with COPD following PR and 2) to investigate associations between changes in $\mathrm{RC}$, religiosity and exercise capacity, quality of life (QoL), anxiety, depression, and dyspnea.
\end{abstract}

Methods: Seventy-four patients were enrolled in this study including 38 patients in the PR group and 36 patients in the control group. PR protocol was composed of a 12-week (three sessions per week, 60 min per day) outpatient comprehensive program, and the control group was composed of patients in a waiting list for admission to PR program. RC, religiosity, exercise capacity, QoL, anxiety, depression, and dyspnea were measured before and after the study protocol.

Results: Positive religious coping and organizational religious activities increased ( $p=0.01$; $p<0.001$, respectively), while negative religious coping decreased ( $p=0.03$ ) after 12 weeks in the PR group $(p<0.001)$. Significant associations were observed between changes in $\mathrm{RC}$, organizational religiosity with exercise capacity, and QoL following PR. No differences were found in the control group.

Conclusion: PR improves RC and organizational religiosity in patients with COPD, and these improvements are related to increases in exercise capacity and QoL.

Keywords: religious coping, religiosity, pulmonary rehabilitation, chronic obstructive pulmonary disease

\section{Introduction}

Chronic obstructive pulmonary disease is a preventable and treatable disease characterized by persistent airflow limitation, usually progressive and associated with an increased inflammatory response of the airways and lungs to the inhalation of noxious particles or gases. ${ }^{1}$ Currently, it is considered as the fourth leading cause of mortality worldwide ${ }^{2}$ and according to the predictions, it will become the third leading cause of death by $2020 .^{3}$

COPD is frequently accompanied by extrapulmonary manifestations, such as skeletal muscle dysfunction, wasting, osteoporosis ${ }^{4}$ that culminates in the appearance of dyspnea cough, exercise intolerance, worsening nutritional status, and increased levels of anxiety and depression. ${ }^{5}$ These symptoms are associated with difficulties to cope with the disease limits, family dependence, emotional and social restriction, and the consequent perspective of death's proximity, compromising patients' quality of life (QoL). ${ }^{6}$ 
Thus, strategies to cope with these challenges are very important. ${ }^{7}$ Religious coping (RC) and religiosity are considered coping strategies in several chronic diseases, especially those with predictive of outcomes in mental and physical health, recently receiving increased attention in health research in $\mathrm{COPD}^{8}$ and end-stage pulmonary disease. ${ }^{9} \mathrm{RC}$ is defined as the use of behavioral and cognitive techniques in stressful life events in a multidimensional construct with positive and negative effects on outcomes, while religiosity is considered a use of individual beliefs, values, practices, and rituals related to faith. ${ }^{10}$

Regarding the interventions for the management of COPD, pulmonary rehabilitation (PR) promotes important benefits including improvements in exercise capacity, depressive symptoms, anxiety, QoL, and dyspnea. ${ }^{11}$ A previous study has shown that PR results in coping changes in styles in COPD patients, and these changes are related to improvements in exercise capacity, anxiety, and depression. ${ }^{12}$ However, to our knowledge, no research has been done assessing the $\mathrm{RC}$ and religiosity in patients with COPD before and after a PR program.

The aims of this study were 1) to compare RC and religiosity in patients with COPD following PR and 2) to investigate associations between changes in $\mathrm{RC}$, religiosity and exercise capacity, QoL, anxiety, depression, and dyspnea.

\section{Methods}

\section{Study design and participants}

A nonrandomized controlled clinical trial of $\mathrm{RC}$ and religiosity in COPD patients before and after a PR program was performed. Patients with moderate-to-very severe $\mathrm{COPD}^{1}$ and clinically stable (no hospitalization or infection in the previous 3 months) were admitted to a pulmonary rehabilitation center at a teaching hospital in Fortaleza (northeastern Brazil) for a comprehensive PR program or a control group between January 2014 and December 2016.

All subjects gave their informed written consent before participation, and the study was previously approved by the ethics institutional review board (Comitê de Ética do Hospital de Messejana Dr Carlos Alberto Studart Gomes, Approval Number: 1.107.599).

\section{Assessments}

Sociodemographics and clinical variables (age, gender, marital status, religious affiliation, comorbidities, body composition, and lung function) were recorded during the baseline assessment.
$\mathrm{RC}$, religiosity, exercise capacity, QoL, anxiety and depression levels, depressive symptoms severity, and dyspnea were assessed at baseline and after completion of study protocol.

RC was assessed with the Brief Religious Coping questionnaire (Brief-RCOPE) which is composed of 14 items distinguishing between positive religious coping (PRC) and negative religious coping (NRC) styles: seven items reflect PRC and seven items reflect NRC (15). The score of each item ranges from 1 ("not at all") to 4 ("a great deal"), and the total score ranges from 7 to 28 for each subscale; the higher the score, the stronger the PRC and NRC, respectively. PRC items rely on a secure relationship with God, whereas NRC items reflect religious struggle that grows out of a more tenuous relationship with God. ${ }^{13,14}$

Religiosity was obtained using the Duke University Religion Index (DUREL). DUREL is a 5-item Likert-type scale measuring three dimensions of religiosity: organizational religious activity (ORA) that asks about the frequency of attendance at religious services (masses, cults, ceremonies, groups of studies or prayer); nonorganizational religious activity (NORA) that asks about the frequency of private religious activities (praying, meditating, reading religious texts, listening to or watching religious programs); and intrinsic religiosity (IR) that refers to the search for internalization and full experience of religiosity as the main objective of the individual. The scores ranging from 1 to 6 for ORA and NORA and 1 to 5 for IR. The scoring of the DUREL is particularly important for both analysis purposes and interpretation of results; besides, summing all three "subscales" into a total overall religiosity score is not recommended. ${ }^{15,16}$

Exercise capacity was measured in the 6-min walk test (6MWT), which was performed according to the guidelines established by the European Respiratory Society and American Thoracic Society where the patient was encouraged to walk as much as possible in a 30 -m flat surface corridor for a period of $6 \mathrm{~min}$. At the end of the test, the examiner recorded the distance traveled. ${ }^{17}$

QoL was measured with two validated Brazilian Portuguese version instruments: COPD Assessment Test (CAT) $)^{18}$ and Saint George's Respiratory Questionnaire (SGRQ). ${ }^{19}$ The CAT is a specific instrument for patients with COPD composed of eight items that analyze the impact of disease symptoms with scores ranging from 0 (optimal) to 40 (worst) points. SGRQ is a specific instrument for individuals with respiratory disease and addresses aspects related to three domains: symptoms, activities, and psychosocial impacts. A total score of the three domain scores was provided. 
Each domain has a maximum possible score presenting a final score of $0-100$, in which 0 corresponds to a better QoL and 100 a worse QoL.

Anxiety and depression levels were evaluated through Hospital Anxiety and Depression Scale (HADS), which consists of seven items for anxiety (HADS-A) and seven for depression (HADS-D). Items are scored on a 4-point scale from 0 (not present) to 3 (considerable), and the scores are added, giving subscale scores on the anxiety and the depression from 0 to 21 . A score $>8$ indicates clinically significant symptoms of anxiety or depression. ${ }^{20}$

Depressive symptom severity was assessed using the Patient Health Questionnaire-9 (PHQ-9). The frequency of symptoms is rated on a 0-3 Likert-type scale, and the summed scores range from 0 to 27 , with higher scores indicating more severe symptoms. ${ }^{21}$

Dyspnea was assessed with the modified Medical Research Council (mMRC), which is widely used to measure breathlessness because of brevity and simplicity. ${ }^{22}$

\section{Pulmonary rehabilitation program}

After the baseline assessment, patients participated in a 12-week (three sessions per week, 60 min per day) outpatient comprehensive PR program. The program was performed by an interdisciplinary team including physiotherapist, chest physician, dietician, occupational therapist, psychologist, and social worker. Physical training was composed of warm-up exercises, upper limb (UL) and lower limb (LL) stretching exercises, UL strengthening exercises, and aerobic conditioning on a treadmill at $80 \%$ of peak work capacity determined by endurance test. Nutritional support, psychological counseling and educational sessions about pathophysiology of COPD, smoking cessation, acute exacerbations, role of respiratory medication, and importance of physical activity were performed by the psychosocial team. ${ }^{11,23}$

It is emphasized that the PR program did not include an intervention aimed at changing patients' individual RC styles or religiosity.

The control group was composed of patients in a waiting list for admission to PR program. They were all properly medicated and informed about the importance of physical activity practice in their routine, which was not followed by a multidisciplinary team.

\section{Statistical analysis}

Statistical analysis was performed using SPSS 20.0 (SPSS Inc., Chicago, IL, USA). Data are presented as absolute or relative frequency, or both, represented by the mean $\pm \mathrm{SD}$.
The variation (delta) between before and after the protocol is presented as the mean $(95 \% \mathrm{CI})$. The independent $t$-test was used to compare continuous variables between the two groups at baseline. Paired Student's $t$-test or Wilcoxon test was used to compare between variables in the two groups before and after the protocol. Pearson correlation was used to evaluate the association between the variables. A $p$-value of $<0.05$ was considered statistically significant. A Bonferroni correction for multiple comparisons to alter the $p$-value to a more stringent value was carried out, thus making it less likely to commit Type I error.

\section{Results}

The 74 patients enrolled in this study included 38 patients in the PR group and 36 patients in the control group. Patients of PR and control groups had similar baseline sociodemographics and clinical variables' characteristics (Table 1).

After applying the Bonferroni correction for multiple comparisons, the critical value for an individual test was

Table I Baseline patient characteristics

\begin{tabular}{|c|c|c|c|}
\hline Characteristics & $\begin{array}{l}\text { PR group } \\
(n=38)\end{array}$ & $\begin{array}{l}\text { Control } \\
\text { group } \\
(n=36)\end{array}$ & $p$-value \\
\hline Age (years) ${ }^{\mathrm{a}}$ & $67.7 \pm 6.9$ & $66.1 \pm 7.7$ & 0.3 \\
\hline Gender (male), n (\%) & $20(52.6)$ & $24(66.7)$ & 0.8 \\
\hline Marital status (married), n (\%) & $22(57.9)$ & $18(47.4)$ & 0.08 \\
\hline Religious affiliation (Catholic), $\mathrm{n}(\%)^{\mathrm{b}}$ & $31(81.6)$ & $28(77.8)$ & 0.9 \\
\hline Comorbidities $\mathrm{n}(\%)^{\mathrm{b}}$ & $\mathrm{I}(0-I)$ & $\mathrm{I}(0-1)$ & 0.9 \\
\hline BMI $\left(\mathrm{kg} \mathrm{m}^{2}\right)^{\mathrm{a}}$ & $27.1 \pm 6.3$ & $25 \pm 4.2$ & 0.1 \\
\hline $\mathrm{FEV}_{1}(\% \text { predicted })^{\mathrm{a}}$ & $46.1 \pm 17.4$ & $42.1 \pm 14$ & 0.2 \\
\hline FCV (\% predicted $)^{\mathrm{a}}$ & $69.3 \pm 16.8$ & $63.8 \pm 16.4$ & 0.1 \\
\hline \multicolumn{4}{|l|}{ Religious copinga } \\
\hline PRC (scores) & $27.1 \pm 1.1$ & $27 \pm 1.4$ & 0.8 \\
\hline NRC (scores) & $9.3 \pm 4.9$ & $7.9 \pm 9.2$ & 0.1 \\
\hline \multicolumn{4}{|l|}{ Religiosity ${ }^{\mathrm{a}}$} \\
\hline ORA (scores) & $4.1 \pm 1.5$ & $4.7 \pm 1.2$ & 0.8 \\
\hline NORA (scores) & $5.2 \pm 1.1$ & $5.6 \pm 0.4$ & 0.6 \\
\hline IR (scores) & $13.3 \pm 2.3$ & $13.5 \pm 0.9$ & 0.6 \\
\hline 6MWD $(m)^{a}$ & $377.1 \pm 97.1$ & $340.7 \pm 75.9$ & 0.07 \\
\hline CAT (points) ${ }^{\mathrm{a}}$ & $16.7 \pm 9$ & $19.6 \pm 6.3$ & 0.1 \\
\hline SGRQ total (points) & $54.5 \pm 15.5$ & $54 \pm 17.9$ & 0.8 \\
\hline HADS-A (points) ${ }^{\mathrm{a}}$ & $5.8 \pm 4.2$ & $6.1 \pm 3.8$ & 0.7 \\
\hline HADS-D (points) ${ }^{\mathrm{a}}$ & $5 \pm 3.5$ & $6.8 \pm 4.2$ & 0.2 \\
\hline PHQ-9 (points) ${ }^{\mathrm{a}}$ & $9.5 \pm 6.2$ & $11.3 \pm 6.7$ & 0.2 \\
\hline mMRC Dyspnea Scale (grade) ${ }^{\mathrm{a}}$ & $2.1 \pm 1$ & $2.6 \pm 1.1$ & 0.09 \\
\hline
\end{tabular}

Notes: ${ }^{a}$ Mean \pm SD. ${ }^{b}$ Data expressed as relative frequency.

Abbreviations: 6MWD, 6-min walk distance; BMI, body mass index; CAT, COPD assessment test; $\mathrm{FEV}_{1}$, forced expiratory volume in I s; FVC, forced vital capacity; HADS-A, Hospital Anxiety and Depression Scale - Anxiety Subscale; HADS-D, Hospital Anxiety and Depression Scale - Depression Subscale; IR, intrinsic religiosity; mMRC, modified Medical Research Council; NORA, nonorganizational religious activity; NRC, negative religious coping; ORA, organizational religious activity; PHQ-9, Patient Health Questionnaire-9; PRC, positive religious coping; SGRQ, St George's Respiratory Questionnaire. 
$\alpha=0.04$, and we considered only individual tests with $p<0.04$ being considered significant.

\section{$R C$ and religiosity before and after PR program}

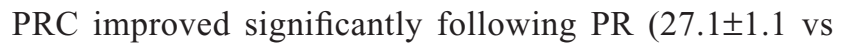
$27.3 \pm 1.1 ; p=0.01)$ as well as NRC $(9.3 \pm 4.9$ vs $8.4 \pm 3.7$; $p=0.03)$. These improvements were not observed in the control group (Table 2).

ORA increased significantly following PR (4.1 \pm 1.5 vs


NORA and IR did not change following PR or control group $(p>0.05)$ (Table 2).

\section{Exercise capacity, QoL, anxiety and depression levels, depressive symptoms severity, and dyspnea before and after PR program}

Following PR, significant improvements were found for the following parameters: 6MWD $(p<0.001)$, CAT scores $(p=0.001)$, SGRQ total score $(p<0.001$, respectively), anxiety/depression levels ( $p<0.001$, respectively), depressive symptom severity ( $p=0.001)$, and dyspnea $(p=0.002)$. These improvements were not observed in the control group (Table 2).

\section{Relationship between changes in RC, religiosity, and PR outcomes}

Changes in PRC following PR were correlated significantly with changes in $6 \mathrm{MWT}(\mathrm{r}=0.36 ; p<0.05)$ and negatively correlated with CAT $(\mathrm{r}=0.36 ; p<0.05)$, while changes in $\mathrm{NRC}$ were inversely correlated with changes in CAT $(\mathrm{r}=0.48$; $p<0.01)$ and PHQ-9 $(\mathrm{r}=0.33 ; p<0.05)$. ORA was correlated with changes in $6 \mathrm{MWT}(\mathrm{r}=0.32 ; p<0.05)$ and negatively correlated with CAT $(\mathrm{r}=-0.46 ; p<0.01)$. Changes in NORA and IR with PR outcomes were not found (Table 3).

There were no significant correlations between changes in $\mathrm{RC}$ and religiosity with anxiety and dyspnea following PR (Table 3).

\section{Discussion}

This study presented the results of the first trial that examined $\mathrm{RC}$ and religiosity in patients with COPD following PR compared to a control group. Important improvements with an increase in PRC and frequency of religious practices and a decrease in NRC were observed. Furthermore, significant associations between $\mathrm{RC}$, organizational religiosity, exercise capacity, and QoL were observed.

As expected, there were significant improvements in physical capacity, QoL, anxiety and depression levels, depressive symptoms severity, and dyspnea after PR. These results are reported well in the literature; ${ }^{11}$ however, to our

Table 2 Changes following PR

\begin{tabular}{|c|c|c|c|c|c|c|c|c|}
\hline \multirow[t]{2}{*}{ Variable } & \multicolumn{4}{|c|}{ PR group $(n=38)$} & \multicolumn{4}{|c|}{ Control group $(n=36)$} \\
\hline & Before & After & $\begin{array}{l}\text { Mean change } \\
\Delta(95 \% \mathrm{Cl})\end{array}$ & $p$-value & Before & After & $\begin{array}{l}\text { Mean change } \\
\Delta(95 \% \mathrm{Cl})\end{array}$ & $p$-value \\
\hline \multicolumn{9}{|l|}{ Religious coping } \\
\hline PRC (scores) & $27.1 \pm 1.1$ & $27.3 \pm 1.1$ & $0.2(0.06,0.5)^{\mathrm{a}}$ & 0.01 & $27 \pm 1.4$ & $27 \pm 1.2$ & $0.02(-0.07,0.1)$ & 0.5 \\
\hline NRC (scores) & $9.3 \pm 4.9$ & $8.4 \pm 3.7$ & $-0.8(-1.6,-0.05)^{\mathrm{a}}$ & 0.03 & $7.9 \pm 2.2$ & $7.9 \pm 2.2$ & $-0.02(-0.08,0.02)$ & 0.3 \\
\hline \multicolumn{9}{|l|}{ Religiosity } \\
\hline ORA (scores) & $4.1 \pm 1.5$ & $5 \pm 1.2$ & $0.8(0.5,1.2)^{\mathrm{a}}$ & $<0.001$ & $4.7 \pm 1.2$ & $4.9 \pm 1.2$ & $0.2(-0.04,0.5)$ & 0.1 \\
\hline NORA (scores) & $5.2 \pm 1.1$ & $5.2 \pm 1.2$ & $0.05(-0.05,0.1)$ & 0.3 & $5.6 \pm 0.4$ & $5.6 \pm 0.4$ & $0.05(-0.1,0.2)$ & 0.4 \\
\hline IR (scores) & $13.3 \pm 2.3$ & $13.5 \pm 2.1$ & $0.1(-0.07,-0.4)$ & 0.1 & $13.5 \pm 0.9$ & $13.6 \pm 0.8$ & $0.02(-0.02,-0.08)$ & 0.3 \\
\hline 6MWD (m) & $377.1 \pm 97.1$ & $421.5 \pm 91.6$ & $44.3(34.6,54.0)^{\mathrm{a}}$ & $<0.001$ & $340.7 \pm 75.9$ & $333.8 \pm 84.3$ & $-6.8(-14.7,0.9)$ & 0.08 \\
\hline CAT (points) & $16.7 \pm 9$ & $14.8 \pm 8.7$ & $-1.8(-2.8,-0.8)^{\mathrm{a}}$ & 0.001 & $19.6 \pm 6.3$ & $19.4 \pm 5.8$ & $-0.1(-0.6,0.2)$ & 0.4 \\
\hline SGRQ total (points) & $54.5 \pm 15.5$ & $36.6 \pm \mid 4.1$ & $-17.8(-21.5,-14)^{\mathrm{a}}$ & $<0.001$ & $54 \pm 17.9$ & $54.8 \pm 16.2$ & $0.8(-0.05,1.8)$ & 0.6 \\
\hline HADS-A (points) & $5.8 \pm 4.2$ & $4.1 \pm 3$ & $-1.7(-2.5,-0.8)^{\mathrm{a}}$ & $<0.001$ & $6.1 \pm 3.8$ & $6.2 \pm 3.7$ & $0.08(-0.1,2.7)$ & 0.3 \\
\hline HADS-D (points) & $5 \pm 3.5$ & $3.8 \pm 3.3$ & $-1.2(-1.8,-0.5)^{\mathrm{a}}$ & $<0.001$ & $6.8 \pm 4.2$ & $6.8 \pm 3.5$ & $-0.02(-0.2,0.2)$ & 0.8 \\
\hline PHQ-9 (points) & $9.5 \pm 6.2$ & $8.1 \pm 5.2$ & $-1.3(-2.5,0.8)^{\mathrm{a}}$ & 0.001 & $11.3 \pm 6.7$ & $11.5 \pm 6.4$ & $0.1(-0.2,0.4)$ & 0.4 \\
\hline mMRC dyspnea scale (grade) & $2.1 \pm 1$ & $1.7 \pm 0.9$ & $-0.4(-2,-0.6)^{\mathrm{a}}$ & 0.002 & $2.6 \pm 1.1$ & $2.5 \pm 1$ & $-0.05(-0.1,0.05)$ & 0.3 \\
\hline
\end{tabular}

Notes: Data are expressed as mean \pm SD and deltas as mean. ${ }^{a} p<0.04$ between groups.

Abbreviations: $\Delta$, mean change; 6MWD, 6-min walk distance; CAT, COPD assessment test; HADS-A, Hospital Anxiety and Depression Scale - Anxiety Subscale; HADS-D, Hospital Anxiety and Depression Scale - Depression Subscale; IR, intrinsic religiosity; mMRC, modified Medical Research Council; NORA, nonorganizational religious activity; NRC, negative religious coping; ORA, organizational religious activity; PHQ-9, Patient Health Questionnaire-9; PR, pulmonary rehabilitation; PRC, positive religious coping; SGRQ, St George's Respiratory Questionnaire. 
Table 3 Associations between changes in RC, religiosity, and outcomes of PR

\begin{tabular}{llllll}
\hline Variable & $\Delta$ PRC & $\Delta$ NRC & $\Delta$ ORA & $\Delta$ NORA & $\Delta$ IR \\
\hline$\Delta$ 6MWD & $0.36^{\mathrm{a}}$ & -0.21 & $0.32^{\mathrm{a}}$ & -0.19 & 0.13 \\
$\Delta$ CAT & $-0.30^{\mathrm{a}}$ & $-0.48^{\mathrm{b}}$ & $-0.46^{\mathrm{b}}$ & 0.18 & -0.16 \\
$\Delta$ SGRQ total & 0.20 & 0.24 & 0.11 & -0.02 & 0.05 \\
$\Delta$ HADS-A & -0.03 & 0.14 & -0.10 & -0.17 & 0.34 \\
$\Delta$ HADS-D & -0.15 & 0.04 & -0.12 & -0.11 & 0.13 \\
$\Delta$ PHQ-9 & -0.26 & $0.33^{\mathrm{a}}$ & -0.18 & -0.20 & 0.38 \\
$\Delta$ mMRC dyspnea scale & -0.14 & 0.11 & 0.05 & -0.21 & -0.03 \\
\hline
\end{tabular}

Notes: ${ }^{a} p<0.05$. ${ }^{b} p<0.01$.

Abbreviations: $\Delta$, mean change; 6MWD, 6-min walk distance; CAT, COPD assessment test; HADS-A, Hospital Anxiety and Depression Scale - Anxiety Subscale HADS-D, Hospital Anxiety and Depression Scale - Depression Subscale; IR, intrinsic religiosity; mMRC, modified Medical Research Council; NORA, nonorganizationa religious activity; NRC, negative religious coping; ORA, organizational religious activity; PHQ-9, Patient Health Questionnaire-9; PR, pulmonary rehabilitation; PRC, positive religious coping; RC, religious coping; SGRQ, St George's Respiratory Questionnaire.

knowledge, the impact of a PR program in RC and religiosity of COPD patients has never been studied.

In the present study, the scores of PRC and ORA increased, whereas the scores of NRC decreased after PR. Previous crosssectional studies have demonstrated that $\mathrm{RC}$ and religious practices are commonly used to cope with numerous chronic illnesses including COPD and are associated with positive clinical outcomes and less progression of diseases. ${ }^{8,9,24-27}$

Pargament ${ }^{10}$ affirms that patients with stronger religious beliefs tend to be more rigorous about their lifestyle changes and adherence of well-being activities, which corroborate with the results of a longitudinal study with patients in post myocardial infarction and post coronary artery bypass surgery (CABS) submitted to a 12-week cardiac rehabilitation program $(\mathrm{CRP})^{28}$ suggesting a development of $\mathrm{RC}$ interventions for cardiac patients and evaluation of the impact of these interventions on medical, spiritual, and psychological outcomes.

There are few studies in the literature which compare changes in PRC and NRC prior to and following interventions using the same instrument of the present study (BriefRCOPE) and none with COPD patients. Piderman et $\mathrm{al}^{29}$ showed improvement in PRC in alcoholic individuals after participating in an outpatient treatment program composed of cognitive-behavioral and motivational enhancement strategies. Bay et $\mathrm{al}^{30}$ found an increase in PRC and a decrease in NRC after chaplain interventions in CABS patients. These results provide evidence that scores on the Brief RCOPE are sensitive to changes after an interventional protocol.

Significant and positive association between changes in NRC and depressive symptoms after PR was found, which corroborates with a previous study with COPD; ${ }^{6}$ however, this mechanism has not yet been properly identified. Horita et $\mathrm{al}^{31}$ affirm that COPD patients with depression make fewer attempts to improve their health, whereas another study ${ }^{32}$ says that they interpret NRC as God's punishment with association of poorer QoL, which corroborates with our findings.

Organizational religiosity improves only in the PR group increasing participation in religious activities, which can be explained by the improvement in patients' functional capacity such as exercise capacity and QoL reducing social isolation and contributing to increase in patients' confidence to leave home and participate in religious activities. ${ }^{33}$ These findings were also reported in cardiac patients submitted to a CRP, ${ }^{28,34}$ which suggests that comprehensive cardiopulmonary rehabilitation programs allow the engagement in religious activities such as frequency of attendance at religious services.

We found significant correlation with changes in organizational religiosity and exercise capacity assessed by the 6MWT, which is an important predictor of severity of disease ${ }^{35}$ because it reflects a greater impairment of the disease when the distance is reduced.

On the other hand, no differences were found in NORA and IR after the PR, which supports the hypothesis that PR improves physical and mental health, but does not have influence on private religious beliefs. Trevino and McConnell ${ }^{28}$ suggest that religious care interventions may not be an appropriate modality in CRP because some patients may not be religious or consider their religious beliefs/practices a personal issue to be dealt outside of their medical management. In our sample, religious discussion did not take part in the PR group. The improvement in RC may be explained by the social interaction element and exercise benefit seen with PR that allowed individuals to take part in religious practices.

Nevertheless, Blinderman et $\mathrm{al}^{6}$ affirm that religiosity does not play a definite role in healthcare decisions, but the evaluation of religiosity among patients with COPD may help to increase understanding of coping strategies, especially due to the high prevalence of this disease and the impact on patients' QoL worldwide.

\section{Limitations}

The results of this study will contribute to future research with definitive conclusions about the role of $\mathrm{RC}$ and religiosity in COPD patients who participated in a PR program; however, our findings should be better elucidated. This study was not randomized as it would be unethical to do random allocation because PR is considered a standard intervention in COPD. The control group was composed of patients who 
were on the waiting list for PR matched to sociodemographic variables and pulmonary function.

In addition, this research was performed at a northeast region of Brazil using a convenience sample, which limits generalizations to other locations. Furthermore, the study population consisted of Roman Catholic individuals, which limits the application of our findings to other populations; thus, we strongly recommend additional researches with more diverse samples to expand our findings, increase knowledge in the studied area, and evaluate the effects of including religious/spiritual care in PR programs.

\section{Conclusion}

In conclusion, our results demonstrate that $\mathrm{PR}$ improves $\mathrm{RC}$ and organizational religiosity in patients with COPD, and these improvements are related to increases in exercise capacity and QoL.

\section{Author contributions}

GPFS - conception and design of the project, acquisition of data, understanding and interpreting the data, drafting and revising the article; FABN - conception and design of the project, acquisition of data, drafting and revising the article; TPMM and MTM - conception and design of the project, acquisition of data, drafting the article; RM and EDBP conception and design of the project, understanding and interpreting the data, drafting and revising the article.

\section{Disclosure}

The authors report no conflicts of interest in this work.

\section{References}

1. Vogelmeier CF, Criner GJ, Martinez FJ, et al. Global strategy for the diagnosis, management, and prevention of chronic obstructive lung disease 2017 report: GOLD executive summary. Eur Respir J. 2017; 49(3): 1700214.

2. Lozano R, Naghavi M, Foreman K, et al. Global and regional mortality from 235 causes of death for 20 age groups in 1990 and 2010: a systematic analysis for the Global Burden of Disease Study 2010. Lancet. 2012;380(9859):2095-2128.

3. Chen JC, Mannino DM. Worldwide epidemiology of chronic obstructive pulmonary disease. Curr Opin Pulm Med. 1999;5(2):93-99.

4. Wouters EF. Chronic obstructive pulmonary disease 5: systemic effects of COPD. Thorax. 2002;57(12):1067-1070.

5. Barnes PJ, Celli BR. Systemic manifestations and comorbidities of COPD. Eur Respir J. 2009;33(5):1165-1185.

6. Blinderman CD, Homel P, Billings JA, Tennstedt S, Portenoy RK. Symptom distress and quality of life in patients with advanced chronic obstructive pulmonary disease. J Pain Symptom Manage. 2009;38(1): $115-123$.

7. Ano GG, Vasconcelles EB. Religious coping and psychological adjustment to stress: a meta-analysis. J Clin Psychol. 2005;61(4):461-480.

8. Green MR, Emery CF, Kozora E, Diaz PT, Make BJ. Religious and spiritual coping and quality of life among patients with emphysema in the National Emphysema Treatment Trial. Respir Care. 2011;56(10): $1514-1521$
9. Burker EJ, Evon DM, Sedway JA, Egan T. Religious coping, psychological distress and disability among patients with end-stage pulmonary disease. J Clin Psychol Med S. 2004;11(3):179-193.

10. Pargament KI. The Psychology of Religion and Coping: Theory, Research, Practice. New York, NY: Guilford Press; 1997.

11. Spruit MA, Singh SJ, Garvey C, et al; ATS/ERS Task Force on Pulmonary Rehabilitation. An official American Thoracic Society/European Respiratory Society statement: key concepts and advances in pulmonary rehabilitation. Am J Respir Crit Care Med. 2013;188(8):e13-e64.

12. Stoilkova A, Janssen DJ, Franssen FM, Spruit MA, Wouters EF. Coping styles in patients with COPD before and after pulmonary rehabilitation. Respir Med. 2013;107(6):825-833.

13. Pargament K, Feuille M, Burdzy D. The brief RCOPE: current psychometric status of a short measure of religious coping. Religions. 2011; 2(1):51-76.

14. Panzini RG, Bandeira DR. Escala de coping religioso-espiritual (Escala CRE1): elaboração e validação de construto. Psicol Estud. 2005; 10(3):507-516.

15. Koenig HG, Büssing A. The Duke University Religion Index (DUREL): a five-item measure for use in epidemiological studies. Religions. 2010;1(1):78-85.

16. Taunay TC, Gondim F de A, Macêdo DS, et al. Validity of the Brazilian version of the Duke Religious Index (DUREL). Rev Psiquiatr Clin. 2012;39(4):130-135.

17. Holland AE, Spruit MA, Troosters T, et al. An official European Society/ American Thoracic Society technical standard: field walking tests in chronic respiratory disease. Eur Respir J. 2014;44(6):1428-1446.

18. Silva GPF, Morano MTAP, Viana CMS, Magalhães CBA, Pereira EDB. Validação do Teste de Avaliação da DPOC em português para uso no Brasil. J Bras Pneumol. 2013;39(4):402-408.

19. Sousa TC, Jardim JR, Jones P. Validação do Questionário do Hospital Saint George na Doença Respiratória (SGRQ) em pacientes portadores de doença pulmonar obstrutiva crônica no Brasil. J Pneumol. 2000; 26(3):119-128.

20. Botega NJ, Bio MR, Zomignani MA, Garcia C, Pereira WA. Mood disorders among medical in-patients: a validation study of the Hospital Anxiety and Depression Scale (HAD). Rev Saude Publica. 1995; 29(5):355-363.

21. Santos IS, Tavares BF, Munhoz TN, et al. Sensibilidade e especificidade do Patient Health Questionnaire-9 (PHQ-9) entre adultos da população geral. Cad Saúde Pública. 2013;29(8):1533-1543. Portuguese.

22. Sociedade Brasileira de Pneumologia e Tisiologia. II Consenso Brasileiro de Doença Pulmonar Obstrutiva Crônica (DPOC) - 2004. J Bras Pneumol. 2004;30(5):1-42.

23. Spruit MA, Vanderhoven-Augustin I, Janssen PP, Wouters EF. Integration of pulmonary rehabilitation in COPD. Lancet. 2008;5371(9606): $12-13$.

24. Strada EA, Homel P, Tennstedt S, Billings JA, Portenoy RK. Spiritual well-being in patients with advanced heart and lung disease. Palliat Support Care. 2013;11(3):205-213.

25. King M, Speck P, Thomas A. The effect of spiritual beliefs on outcome from illness. Soc Sci Med. 1999;48(9):1291-1299.

26. Lucchetti G, de Almeida LG, Lucchetti AL. Religiousness, mental health, and quality of life in Brazilian dialysis patients. Hemodial Int. 2012;16(1):89-94.

27. Taheri-Kharame Z, Zamanian H, Foroozanfar S, Afsahi S. Religious wellbeing as a predictor for quality of life in Iranian hemodialysis patients. Glob J Health Sci. 2014;6(4):261-269.

28. Trevino KM, McConnell TR. Religiosity and spirituality during cardiac rehabilitation: a longitudinal evaluation of patient-reported outcomes and exercise capacity. J Cardiopulm Rehabil Prev. 2015;35(4):246-254.

29. Piderman KM, Schneekloth TD, Pankratz VS, Maloney SD, Altchuler SI. Spirituality in alcoholics during treatment. Am J Addict. 2007;16(3): 232-237.

30. Bay PS, Beckman D, Trippi J, Gunderman R, Terry C. The effect of pastoral care services on anxiety, depression, hope, religious coping, and religious problem solving styles: a randomized controlled study. J Relig Health. 2008;47(1):57-69. 
31. Horita N, Kaneko T, Shinkai M, et al. Depression in Japanese patients with chronic obstructive pulmonary disease: a cross-sectional study. Respir Care. 2013;58(7):1196-1203.

32. Pedersen HF, Pargament KI, Pedersen CG. Religious coping and quality of life among severely ill lung patients in a secular society. Int J Psychol Relig. 2013;23(3):188-203.

33. Benjamins MR, Musick MA, Gold DT, George LK. Age-related declines in activity level: the relationship between chronic illness and religious activities. J Gerontol B Psychol Sci Soc Sci. 2003;58(6):S377-S385.
34. Trevino KM, McConnell TR. Religiosity and religious coping in patients with cardiovascular disease: change over time and associations with illness adjustment. J Relig Health. 2014;53(6):1907-1917.

35. Dajczman E, Wardini R, Kasymjanova G, Préfontaine D, Baltzan MA, Wolkove N. Six minute walk distance is a predictor of survival in patients with chronic obstructive pulmonary disease undergoing pulmonary rehabilitation. Can Respir J. 2015;22(4):225-229.

International Journal of COPD

\section{Publish your work in this journal}

The International Journal of COPD is an international, peer-reviewed journal of therapeutics and pharmacology focusing on concise rapid reporting of clinical studies and reviews in COPD. Special focus is given to the pathophysiological processes underlying the disease, intervention programs, patient focused education, and self management protocols.

\section{Dovepress}

This journal is indexed on PubMed Central, MedLine and CAS. The manuscript management system is completely online and includes a very quick and fair peer-review system, which is all easy to use. Visit http://www.dovepress.com/testimonials.php to read real quotes from published authors.

Submit your manuscript here: http://www.dovepress.com/international-journal-of-chronic-obstructive-pulmonary-disease-journal 